\title{
Application of Radio Frequency Identification Positioning Technology in Valuables Monitoring
}

\author{
Guicheng Shen, Kunlun Wang, Chaofan Su, Juntao Li \\ School of information, Beijing Wuzi University, Beijing, China \\ Email address: \\ shenguicheng@bwu.edu.cn (Guicheng Shen), 1148202814@qq.com (Kunlun Wang),874755291@qq.com (Chaofan Su), \\ 862974337@qq.com (Juntao Li) \\ To cite this article: \\ Guicheng Shen, Kunlun Wang, Chaofan Su, Juntao Li. Application of Radio Frequency Identification Positioning Technology in Valuables \\ Monitoring. International Journal of Intelligent Information Systems. Vol. 5, No. 4, 2016, pp. 55-59. doi: 10.11648/j.ijiis.20160504.12
}

Received: May 3, 2016; Accepted: May 13, 2016; Published: August 1, 2016

\begin{abstract}
With the development of positioning services, an increasing number of people and companies pay attention to the management and protection of the precious goods which they possess, through the determination of its location to achieve the management purpose [1]. At present, the main positioning way is to use the global positioning system (GPS), but many of the valuable items are stored in the indoor, and it's hard to receive a satellite signal in the room, so it cannot be accurate positioning. As a new short distance wireless data recognition technology at present, Radio Frequency Identification (RFID) indoor positioning technology is very suitable for the indoor positioning. Because it has many characteristics: large amount of data storage, readable and writable, strong penetrating power, long recognition distance, fast read-write speed and the use of the characteristics of long service life and good adaptability to the environment, etc. Among the RFID indoor positioning technology of many positioning algorithms, the three sides positioning algorithm is commonly used, and more mature. At the same time, the storage space of most valuable items is simple, and the environmental factors have little influence on the signal interference. So it is feasible to use the three sides positioning algorithm of RFID positioning algorithms to locate the valuables in the indoor.
\end{abstract}

Keywords: RFID Technology, Valuables, Monitoring System, Positioning Algorithm

\section{Introduction}

Currently, valuables supervision technology often focused on video monitoring, infrared sensor and magnetic induction door etc. And the monitoring approaches are divided into two types: active and passive. The existing monitoring equipment has its own merits, but when applied to the supervision of the valuables, obvious weaknesses could be found, for instance, video surveillance information is huge, transmission and storage cost is high, and the monitoring method is passive, what's more, it doesn't has the abnormal event detection and alarm function, and needs specialized personnel uninterrupted patrol monitor; And infrared monitoring is largely affected by the environment, monitored infrared can be avoid, criminals who master this technology can easily steal the monitored items; The magnetic induction door is mainly used for monitoring the people who past entrance.

In recent years, with the continuous development of information technology, wireless Radio Frequency
Identification (RFID), which as the bottom of the Internet of things information collection equipment, is becoming more and more mature. This technology has been widely used in many fields, such as logistics warehouse, intelligent transportation, retail and the automatic production line etc. RFID technology has many characteristics: large amount of data storage, read and write, strong penetrating power and long distance of read- write, fast reading speed, long service life and good adaptability to the environment etc. And it is the only automatic recognition technology that can realize the simultaneous identification of multiple targets [2].

Put RFID technology into valuables monitoring and management, not only can improve work efficiency of items management, but also can make the location of the valuables more intuitive, more safety assurance, and also can save part of manpower and material resources. And then realize the automation of the management of valuable goods, which also meet the current trends of intelligent management of goods. It is an important means to achieve standardized management of goods [3]. 
In the indoor environment, because of the presence of walls and ground reflection, furniture, facilities, and personnel to move around, so that the wireless communication becomes very complex. Signal is disturbed by the environment, will produce multipath phenomenon, and when the direct path is blocked, it will produce more serious non line of sight, which has the greatest impact on the RFID tag positioning system. In order to solve this problem, in literature [4] using multi antenna in RFID system, using Mi Mo technology to achieve accurate fault location, so as to improve the accuracy of positioning; literature [5] proposed a modified sub phase method to enhance the robustness of the positioning system. In addition to the accuracy of the measurement can be improved. We can also improve the positioning algorithm to improve the positioning accuracy.

Among RFID indoor positioning technology of many positioning algorithms, the three sides positioning algorithm is commonly used, and more mature $[6,9,10]$. Based on the original three side positioning algorithm, this paper proposes an improved scheme, which is more suitable for location management of indoor valuables.

\section{Correlation Theory}

\subsection{Source of RFID Technology}

RFID (Radio Frequency Identification) technology, also known as wireless Radio Frequency Identification, is a kind of communication technology. A specific target and read -write data can be identified by the radio signal, without the need to establish a mechanical or optical contact between the system and the specific target.

In the actual application process, according to the different application purposes and application environment, the composition of RFID system will vary, but from the working principle of RFID system, in general, the system is composed of by several parts, such as the signal transmitter (general as electronic label), signal receiver (Reader), transmitting and receiving antenna, its working principle is shown in Figure 1:

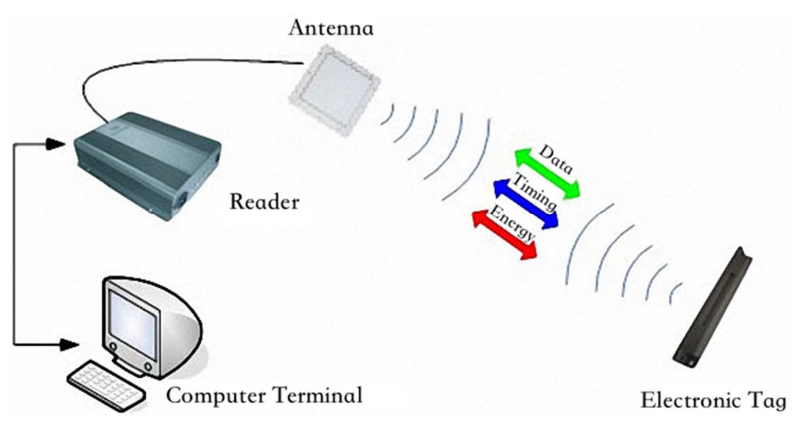

Figure 1. RFID system working principle diagram.

The reader sends a certain frequency of radio frequency signals through the antenna, when the electronic tag which is attached to the target object went into the signal range, it will initiative issue the relevant information that stored in the active electronic tag to reader. After the reader receiving and decoding, it is sent to the central information system by the way of wireless transmission [6].

\subsection{RFID Positioning Principles}

From the basic ideas, RFID positioning principle and common positioning (such as GPS positioning) are basically the same idea. The core technology of positioning is actually distance measurement. If we know the specific coordinates of some points related to the unknown point in space, the coordinates of unknown points can be calculated by a certain positioning algorithm. Pahlavan and his friends proposed wireless positioning system framework [6] which including: location detection device, positioning algorithm and terminal display system. These three parts are shown in figure 2:

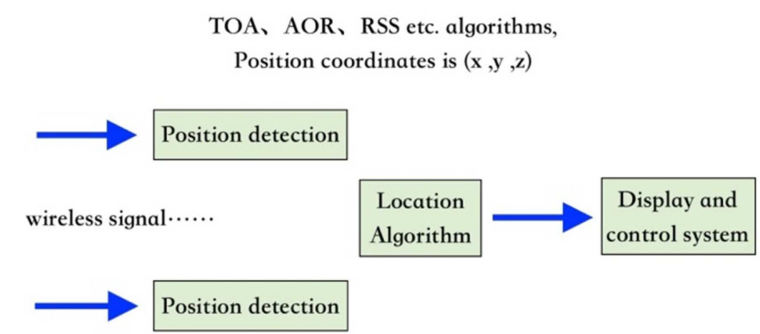

Figure 2. Wireless positioning system framework.

In this thesis, all the valuables in the storeroom are attached with electronic tags, and the reader and the back-end server can achieve wired or wireless connections. Using the reader monitoring the electronic tags, to realize the monitoring and management of the valuables.

\subsection{RFID Positioning Algorithm and Its Application}

RFID wireless signal propagation in different environments, often subject to reflection, diffraction, multipath and non line of sight and other factors of multiple effects. Currently in two-dimensional positioning, the three sides positioning algorithm is commonly used. By the common sense, if the three reference nodes are not in the same line, the distance between the unknown node and the reference nodes can only determine the position of the unknown node. However, in the actual process of positioning, measurement error is inevitable, so three circles formed by the distance of unknown nodes and three reference nodes could not accurately over to a point, that is to say, it is impossible to accurately determine the position of the unknown node, as is shown in Figure 3 [8]:

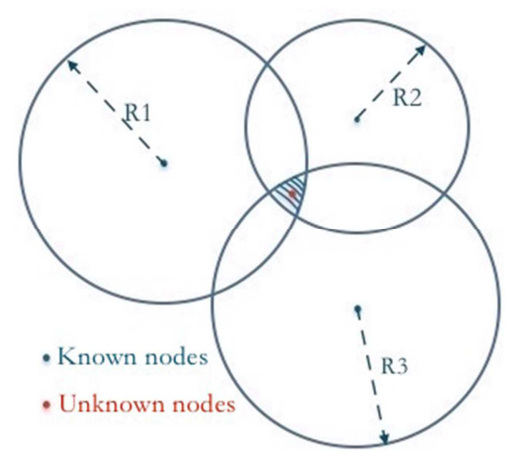

Figure 3. Three sides positioning effect chart 
Take the reference node as the center of a circle, the shadow region above is formed by the three measurement radius $\mathrm{R} 1$, $\mathrm{R} 2, \mathrm{R} 3$ read-write device. Solving the equation, the range of the solution is in the shadow region. The smaller the shadow area, the more accurate the solution will be. Therefore, many scholars have proposed an improved three sides positioning algorithm. Among them, six scholars included the Peigang Sun, Hai Zhao, Guangjie Han [9] proved that when the three reference nodes were placed in an equilateral triangle, the positioning error can reach the minimum.

In this paper, we use this improved three sides positioning algorithm to position and supervise the valuables in the storeroom. The specific algorithm is shown as follows.

As we all know, RFID radio frequency signal propagation speed can be expressed by the approximation of the speed of light $\left(\mathrm{c}=3 \times 10^{8} \mathrm{~m} / \mathrm{s}\right)$. Thus the calculation formula of distance can be indicated as $\mathrm{Ri}=(\mathrm{ti}-\mathrm{t} 0) \times \mathrm{c}$, as is shown in Figure 4 . According to the principle of proximity, we can set three reference points (A, B, C and A, B, C are just form an equilateral triangle) in the nearest position of the target $P$, calculating the position of the targe [7].

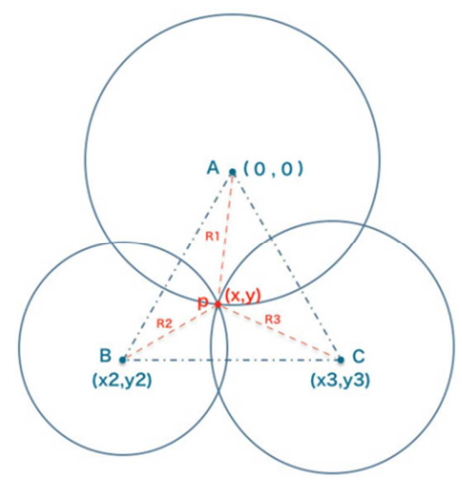

Figure 4. Positioning diagram.

The specific calculation process is given below. First, calculate the distance of the target $\mathrm{P}$ to three reference points $(\mathrm{A}, \mathrm{B}, \mathrm{C})$ :

$$
\left\{\begin{array}{c}
R_{1}^{2}=x^{2}+y^{2} \\
R_{2}^{2}=\left(x_{2}-x\right)^{2}+\left(y_{2}-y\right)^{2} \\
R_{3}^{2}=\left(x_{3}-x\right)^{2}+\left(y_{3}-y\right)^{2}
\end{array}\right.
$$

Then, the coordinates of the target $\mathrm{P}$ can be calculated by using the matrix operation:

$$
\left[\begin{array}{ll}
\mathrm{x}_{2} & \mathrm{y}_{2} \\
\mathrm{x}_{3} & \mathrm{y}_{3}
\end{array}\right]\left[\begin{array}{l}
x \\
y
\end{array}\right]=\frac{1}{2}\left[\begin{array}{l}
K_{2}^{2}-R_{2}^{2}+R_{1}^{2} \\
K_{3}^{2}-R_{3}^{2}+R_{1}^{2}
\end{array}\right]
$$

And, $\mathrm{K}_{\mathrm{i}}{ }^{2}=\mathrm{R}_{\mathrm{i}}{ }^{2}+\mathrm{x}_{\mathrm{i}}{ }^{2}$

The above algorithm can be applied to the control of valuables in the storeroom. The research found that under the general circumstances, the effective area of the valuables storeroom is about $40 \mathrm{~m}^{2}$. We can install 3 sets of static RFID reader in the indoor. In order to ensure that the positioning error is minimized under this algorithm, we will put these three sets of the readers in an equilateral triangle's vertex. Each reader is connected to the back-end management system in a wired way. Through the positioning algorithm and the relative position between the 3 readers and the electronic tag on the goods, we can achieve the valuables monitoring, including to make timely feedback of the valuables in and out of storeroom, ectopic, and be stolen, etc. As the position of each goods is generally determined, and the position of each goods was collected in advance in the system database, if the positioning system found goods ectopic, move or beyond the reader reading area (a non normal outbound), the data will be immediately transmitted to the management system through the wired network and make the corresponding prompt or alarm.

\section{Design and Implementation of Valuables Monitoring System and Positioning Algorithm Simulation}

\subsection{System Design}

The design of valuables system should follow the following principles: 1) Security; 2) Reliability; 3) Practicability; 4) extendibility; 5) Progressiveness [2].

Through research we found that traditional valuables monitoring system is generally aimed at the management of the goods in the static condition in the indoor and the simple registration of out of the storeroom. But failure to achieve the function of the goods in and out of storage or anti-theft monitoring or alarm processing in the storeroom. Therefore, in the simulation, we add the position management and alarm processing of the goods in the database, through the RFID technology and the positioning algorithm in 2.3 , we can achieve the dynamic monitoring of the valuables storage process and the anti-theft.

The following chart is the RFID valuables dynamic monitoring system design schematic, due to space limitations, there is no detailed visual system interface. The system consists of three modules: system management, valuables database management, configuration and maintenance, each module has a number of corresponding sub modules, as is shown below:

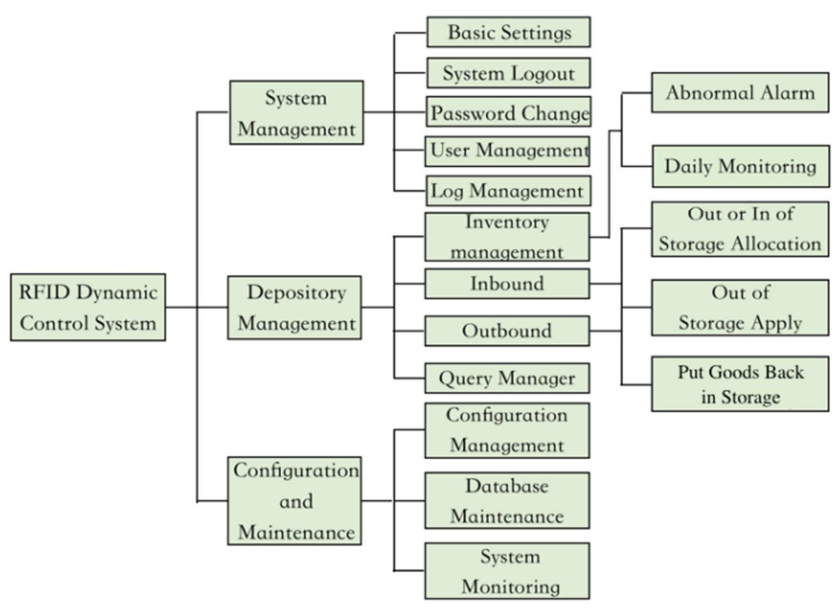

Figure 5. RFID valuables dynamic monitoring system design schematic. 


\subsection{Positioning Algorithm Simulation}

In 2.3, we have discussed the application of the three sides positioning algorithm in the valuables management. In our test, we installed 3 readers in a valuables storage which is about 40 $\mathrm{m}^{2}$ and adjust the radius of read-write to $15 \mathrm{~m}$, in this way, it could be completely covered. Now we are aiming to simulate the management of valuables position in indoor, as is described in 2.3, we can install 3 sets of static RFID reader in the storage and put these three sets of the readers in an equilateral triangle's Vertex [10].

The coverage area of the reader is shown in figure 4, among them, $\mathrm{A}(0,0), \mathrm{B}(\mathrm{x} 2, \mathrm{y} 2)$ and $\mathrm{C}(\mathrm{x} 3, \mathrm{y} 3)$ are the settlement points of the 3 readers, whose purpose are monitoring the target goods $\mathrm{P}(\mathrm{x}, \mathrm{y})$.

In the experiment, after we finished the layout of the reader and other hardware and software facilities, first of all, we collect all the position information of the goods. The purpose is to carry out real-time dynamic monitoring of each goods in the information database, whether it is the goods misplacement or doesn't match the goods or the abnormal information in the process of monitoring, we can obtain all the real-time information and get the prompt message. During the experiment, we also simulated the goods misplacement. If any goods are misplaced in the storeroom, the system will immediately show red, indicating that the position of the goods is incorrect, and at the same time voice prompts "dislocation, please reset!" The process is shown in the following Figure 6:

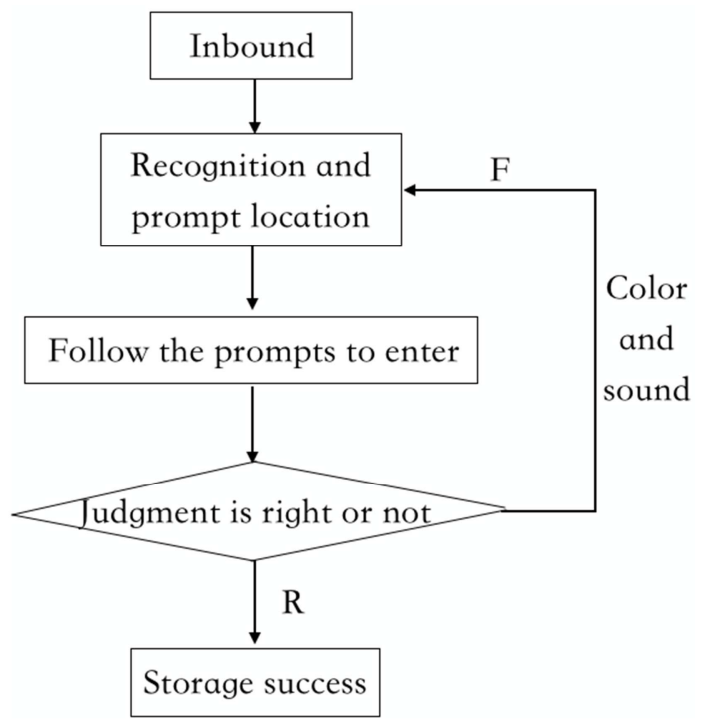

Figure 6. Simulation of goods misplacement.

In the experiment, we further simulated the phenomenon of the vaulables away from the storeroom without any reason. We put the goods, which with electronic tags and has been already registered in the storeroom, out of the storeroom (there is no requirement of whether it is in the covered range of the reader). As shown in Figure 7, it is the reconstructed scene in Cartesian coordinates, A, B, C are the dispatched points of the 3 readers and $\mathrm{M}$ is the position point of the test goods.

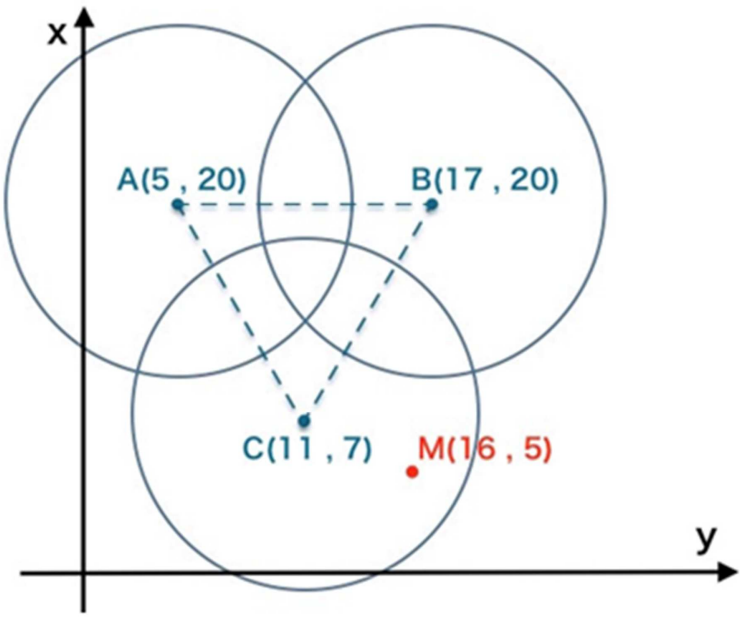

Figure 7. Simulation coverage chart.

It can be seen that $\mathrm{M}$ is in one of the reader's coverage but it's out of the coverage of the other two readers. According to our algorithm description, at this time, it should be unable to locate, and then the system will recognize the goods is not in the storage and disappeared without a trace. Then, the system will automatically pop up a dialog box prompt and alarm. In fact, at this point the reader $\mathrm{C}$ is able to identify the goods $\mathrm{M}$, indicating that the goods are out of the storage but still in the vicinity, when this situation occurs, the system will also make the corresponding prompt.

\section{Conclusion}

Based on the field investigation, this paper analyzed the existing problems of valuables management system, and puts forward the corresponding solution measures. Compared with other research, the innovation of this paper lies in the application of RFID technology in the real-time monitoring and management of valuables. On the basis of ensuring the static management of goods in the past, the real-time dynamic monitoring of the goods is realized, the manpower and material resources are reduced, and the informatization and automation of the monitoring system are improved.

The positioning algorithm mentioned in this paper may not be the best algorithm. To fully realize the immediately reminder of the mistaken and misplacement of the valuables may require more accurate algorithm.

In this paper, based on RFID technology, the research on the dynamic monitoring system of valuables is lack of practicality, whether in the structural design, or in the realization of the function, the actual situation may be considered insufficient. We clearly know that in practical applications, the dynamic monitoring of valuables must have a lot of situations and unexpected events that we can't predict, the content of the study still needs to be verified and improved in practice.

\section{Acknowledgements}

This paper is supported by the Funding Project for Technology Key Project of Municipal Education Commission 
of Beijing (ID: TSJHG201310037036); Funding Project for Beijing Intelligent Logistics System Collaborative Innovation Center; Funding Project for Beijing key laboratory of intelligent logistics system; Funding Project of Construction of Innovative Teams and Teacher Career Development for Universities and Colleges Under Beijing Municipality (ID: IDHT20130517), and Beijing Municipal Science and Technology Project (ID: Z131100005413004); Funding Project for Beijing philosophy and social science research base specially commissioned project planning (ID: 13JDJGD013).

\section{References}

[1] Danshi Sun, Li Yang. ZigBee Positioning Technology in Valuables Monitoring [J]. Geomatics \& Spatial Information Technology, 2015, 07: 191-192.

[2] Xiuli Ma, Xiulian Wang, Libo Wei. Design of monitoring and management system for valuables based on RFID [J]. Automatic identification technology of China, 2009, 01: 21-24.

[3] Desheng Zhang. Application of RFID technology in the management of firearms [J]. National Defense Technology Foundation. 2008, 11: 41-44.
[4] Arnitz D, Reynolds M S. Multitransmiter wireles power transfer optimization for backscater RFID transponders [J]. Antennas and Wireles Propagation Leters, IEEE, 2013, 12 (4): 849-852.

[5] Li X, Zhang Y, Amin M G. Multifrequency-based range estimation of RFID tags [C]//Proc of 2009IEEE International Conference on RFID, IEEE, 2009: 147-154.

[6] Yu Shu, Jiang Wu, Donghui Li, Huiying Kuang. Research on the application of active RFID location algorithm in firearms control system [J]. Mobile Communication. 2015, 10: 61-65.

[7] K. Pahlavan, X. Li, and J. P. Makela. Indoor geolocation science and technology. IEEE Commun. Mag., vol. 40, no. 2, pp. 112-118, Feb. 2002.

[8] Junmin Bai. Research and design of firearms dynamic monitoring system based on RFID Technology [D]. Xi'an Electronic Technology University. 2014

[9] Wenjian Wu, Jin Liu, Helin Li, Bing Kong. An improved weighted three edge location algorithm [J]. Journal of Zhengzhou University of Light Industry (NATURAL SCIENCE EDITION). 2012, 03: 83-85.

[10] Peigang Sun, Hai Zhao, Guangjie Han, Xiyuan Zhang, Jian Zhu. Chaotic triangular positioning reference point selection algorithm [J]. Computer Research and Development. 2007, 12: 1987-1995. 